\title{
绵羊tnp2基因的克隆及其在体外共培养系统中 圆形精子细胞的转录分析
}

\author{
白音巴图，罗奋华，胡甜园，侯 越，吴应积* \\ (内蒙古大学 哺乳动物生殖生物学及生物技术教育部重点实验室, 内蒙古 呼和浩特 010021)
}

\begin{abstract}
摘要: 过渡蛋白 2 基因 (tnp2) 是圆形精子细胞特异表达的基因。为了开展绵羊圆形精子细胞标记基因的研究, 根据GenBank上已公布的牛的cDNA序列设计引物, 采用RT-PCR和分子克隆方法, 克隆了蒙古绵羊tnp2基因cDNA 部分编码区序列。DNA 序列测定结果与牛的核苷酸序列比对, 同源性为 $95.3 \%$ 。根据绵羊tnp2基因的cDNA序列 设计引物, 对共培养的四月龄绵羊睪丸生殖细胞进行RT-PCR 鉴定。结果显示体外共培养的绵羊睪丸生殖细胞一 直到第十周后仍有圆形精子细胞产生。绵羊tnp2基因的cDNA克隆和序列测定为进一步研究绵羊精子发生过程奠 定了基础。
\end{abstract}

关键词: 绵羊; tnp2基因; 克隆; 序列分析; 圆形精子细胞

中图分类号: Q593.4; Q954.4; Q959.842 文献标识码: A 文章编号: 0254-5853-(2009)03-0262-05

\section{Cloning of Sheep tnp2 Gene and Transcription Analysis of Round Spermatid Cells in the in vitro Co-culture System}

\author{
BAI Yin-ba-tu, LUO Fen-hua, HU Tian-yuan, HOU Yue, WU Ying-ji* \\ (The Key Laboratory for Mammalian Reproductive Biology and Biotechnology, Ministry of Education, Inner Mongolia University, \\ Inner Mongolia Hohhot 010021, China)
}

\begin{abstract}
The transition protein-2 gene (tnp2) have been reported as the round spermatid-specific marker gene. However, the DNA sequence of tnp2 gene in Ovis aries have not been reported. In order to study the sheep round spermatid-specific marker gene, we designed primers according to the reported Bos taurus tnp2 gene cDNA sequences in the GenBank. The partial CDS was amplified by RT-PCR. The PCR fragment was inserted into the T-A cloning vector pMD19-T. The partial nucleotide sequences of Mongolia sheep tnp2 gene were compared with the counterpart sequences of Bos taurus, and the nucleotide homology was $95.3 \%$. Using the primers designed according to the tnp2 cDNA sequence, the tnp2 gene transcription expression in the co-cultured cells derived from four-months old sheep testis was assessed by RT-PCR. The results demonstrate that the round spermatid cells are generated in the co-culture system until ten weeks in vitro. The cDNA cloning and sequencing lay down a foundation for further study on spermatogenesis of sheep.
\end{abstract}

Key words: Sheep; tnp2 gene; Cloning; Sequence analysis; Round spermatid

哺乳动物的精子发生是一个在曲细精管内非 常复杂的生殖细胞发育过程。精子发生过程中, 二 倍体精原细胞通过有丝分裂分化成精母细胞, 接着 精母细胞通过减数分裂产生单倍体精子细胞, 随后 精子细胞进行形态分化产生精子(Parvinen, 1982)。 精子发生过程不仅仅受LH (luteinizing hormone) 和
FSH (follicle-stimulating hormone) 等促性腺激素调 控, 同时也受精原细胞和体细胞相互作用的调控 (Skinner, 1991; Kierszenbaum, 1994; Griswold, 1995), 然而具体机制还不是十分清楚。近些年, 生 殖细胞体外培养技术的发展为精子发生过程的体 外重演提供了可能, 使研究者便于通过体外培养的

收稿日期：2009-02-06；接受日期：2009-05-11

基金项目: 内蒙古自治区自然科学基金重大项目 (2004080204) [Natural Science Foundation of Inner Mongolia Municipality in China(2004080204)]

*通讯作者(Corresponding author), 男, 教授, 博士生导师, E-mail:yingji_wu@yahoo.com

第一作者简介: 白音巴图(1983-), 男, 在读硕士, 研究方向为哺乳动物生殖生物学及生物技术 
方法来鉴别生殖细胞分化、减数分裂和精子发生过 程中起作用的因子和基因等。

初级精母细胞分裂产生单倍体的圆形精子细 胞，标志着精子变态过程的开始。精子变态经历了 复杂的形态、生化和生理变化, 这些变化都伴随着 阶段特异性基因表达。圆形精子细胞和其后的分化 形态的鉴定对这一过程的研究具有重要意义。但是 至今为止还未见用于鉴定圆形精子细胞的抗体, 使 得体内外圆形精子细胞相关的研究缺乏可靠的依 据。只从形态特征判定圆形精子细胞是不够精确 的。过渡蛋白 2 基因 (transition protein-2 gene, tnp2) 是在雄性生殖细胞减数分裂后期表达的一个基因, 其 mRNA 最先在圆形精子细胞中合成并储存, 直至 在长型精子细胞中被激活指导翻译(Hecht, 1990; Marret et al, 1998)。因此, 可通过检测 tnp2 基因的 转录表达来判断雄性生殖细胞体外共培养体系中 圆形精子细胞的存在。目前已成功克隆了人、牛、 猪、黑猩猩、小鼠和大鼠的 tnp2 基因, 但绵羊的 tnp2 基因尚未报道。为了进一步研究绵羊的精子发生过 程, 本研究克隆了绵羊 tnp2 基因的 cDNA 部分编码 区, 为圆形精子细胞特异表达基因的转录表达分析 提供了条件。同时通过 RT-PCR 法对我们建立的绵 羊雄性生殖细胞体外共培养体系中 tnp2 基因的转 录表达进行分析, 来检测该体系中是否有圆形精子 细胞的形成, 从分子水平上对体外培养的圆形精子 细胞进行鉴定, 以期为进一步研究绵羊体外精子发 生过程打下基础。

\section{1 材料与方法}

\section{1 试剂及耗材}

pMD19-T载体、限制性内切酶和T4 DNA连接 酶、M-MLV反转录酶、ExTaq DNA聚合酶和DNA Marker等均购自 TakaRa公司, 总RNA提取试剂盒 (Cat.NO.DP419)购自北京天根公司, 胶回收试剂盒 (Cat.NO.28704)购自QIAGEN 公司, E.coli DH5 $\alpha$ 感 受态细胞为本实验室保存。其它化学试剂为国产分 析纯。

\section{2 试验材料}

成年蒙古绵羊睪丸取自于内蒙古穆斯林屠宰

场, 带回实验室后置于 $-80^{\circ} \mathrm{C}$ 冰箱保存备用。

\section{3 睪丸组织的总 RNA的制备}

从绵羊睪丸组织块分离总 RNA。利用 TIANGEN公司的总RNA提取试剂盒按照使用说明
书的操作程序提取绵羊睪丸组织总RNA, 用紫外分 光光度仪测定RNA浓度后, 置于 $-80^{\circ} \mathrm{C}$ 冰箱保存备 用。

\subsection{RT-PCR扩增}

利用M-MLV反转录酶并按照使用说明书的操 作程序进行反转录反应, 得到 $\mathrm{CDNA}$ 第一链。以得 到的 $\mathrm{cDNA}$ 第一链为模板, 利用计算机软件 CLC Free Workbench 4 对已知的人、大鼠、小鼠、牛、 猪和黑猩猩的 tnp 2 基因的核苷酸序列进行同源比 较, 得出tnp2 cDNA序列的保守区。根据保守区中 牛的 tnp2基因 (GenBank accession number:NM 174200)的cDNA编码区序列, 设计一对PCR引物。 上游引物(P1): 5' CCTATGGACACCCAGACTCAC 3'; 下游引物(P2): 5' AGTTGTACTTCCGTCCTGAGC 3'。PCR反应体系为 $10 \mu \mathrm{L}$, 扩增条件为: $94^{\circ} \mathrm{C}$ 预变性 $4 \mathrm{~min} ; 94^{\circ} \mathrm{C}$ 变性 $40 \mathrm{~s}, 56^{\circ} \mathrm{C}$ 退火 $40 \mathrm{~s}, 72^{\circ} \mathrm{C}$ 延伸 $40 \mathrm{~s}$, 共进行 35 个循环; $72^{\circ} \mathrm{C}$ 延伸 $10 \mathrm{~min} 。 \mathrm{Mg}^{2+}$ 浓度通过梯度测试法优化。PCR产物在 $1 \%$ 的琼脂糖 凝胶通过电泳分离，在 $0.01 \%$ 的澳化乙锭 (ethidium bromide, EB) 溶液中染色, 在紫外光激发下通过 凝胶成像仪（Bio-Rad, P91）拍摄图片。

\section{5 cDNA克隆载体的构建与鉴定}

按照 $\mathrm{Mg}^{2+}$ 浓度优化后的条件扩大PCR反应, 然 后利用 QIAGEN胶回收试剂盒回收目的片段 cDNA。测定浓度后, 按照T-A克隆载体pMD19-T 的使用说明书将获得的 cDNA目的片段连接到 pMD19-T克隆载体上, 获得重组DNA。将重组DNA 转化E.coli DH5 $\alpha$ 感受态细胞, 在含有X-gal、IPTG 和氨芐青霉素的 $L B$ 平板培养基中 $37^{\circ} \mathrm{C}$ 培养 $14 \mathrm{~h}$ 后进 行蓝白斑筛选。选择白色菌落, 用质粒提取试剂盒 提取质粒, PCR法篮选阳性质粒, 限制性内切酶 HindIII和BamH1双酶切鉴定重组质粒。将鉴定阳性 的重组质粒送至上海生工生物工程有限责任公司 进行测序。

\section{6 生物信息学方法}

tnp2基因cDNA序列用 NCBI网站上的BLAST (http://www.ncbi.nlm.nih.gov/BLAST/) 进行序列比 对。通过DNAstar(Lasergene7.1)软件推导tnp2基因蛋 白质的氨基酸序列, 并使用 DNAstar分析软件 MegAlign Clustel W (Slow/ Accurate)对绵羊和其他 哺乳动物的tnp2进行cDNA核苷酸序列的同源性比 较。

\subsection{RT-PCR 检测体外培养的生殖细胞中 tnp2基}




\section{因的转录表达}

参照本实验室已建立的睪丸生殖细胞体外培 养方法(Wu et al, 2005; Zhang et al, 2007), 取 4 月龄绵羊睪丸组织, 进行体外共培养。取组织块及 原代共培养期间 2-10 周的细胞, 提取总 RNA, 以 反转录得到的 cDNA 第一链为模板, 以绵羊 gapdh 的编码 $\mathrm{cDNA}$ 为内部参照, 根据本研究克隆的 tnp2 cDNA 编码区序列设计一对引物, 进行 RT-PCR 分 析, 引物如表 1 。同时取绵羊肝脏组织作为转录表 达对照。

\section{2 结 果}

\section{$2.1 \operatorname{tnp} 2$ 基因 $\mathrm{cDNA}$ 的克隆及重组质粒的篮选和} 鉴定

以蒙古绵羊成年睪丸组织总RNA为模板, 经 RT-PCR反应, 得到一个约 $384 \mathrm{bp}$ 的片段(图1), 与预 期的cDNA片段大小相符。胶回收RT-PCR反应扩增 产物, 回收产物与pMD19-T载体(2962bp)连接, 连 接产物转化E.coli DH5a获得阳性克隆。提取质粒 DNA, 用PCR法篮选重组质粒, 经限制性内切酶 BamH I 和HindIII双酶切鉴定。阳性重组质粒经 DNA序列测定结果表明, 插入片段长 $384 \mathrm{bp}$, 经过 序列比对, 证明这个片段确实是绵羊的tnp2基因的 cDNA序列。

表 1 RT-PCR引物

Tab. 1 Oligonucleotides used for RT-PCR

\begin{tabular}{|c|c|c|c|}
\hline $\begin{array}{l}\text { 目的基因 } \\
\text { Target gene }\end{array}$ & $\begin{array}{c}\text { 引物序列 } \\
\text { Sequence of primers }\end{array}$ & $\begin{array}{l}\text { 产物大小 (bp) } \\
\text { Product size }\end{array}$ & $\begin{array}{c}\text { 退火温度 }\left({ }^{\circ} \mathrm{C}\right) \\
\text { Annealing temperature }\end{array}$ \\
\hline tnp2 & $\begin{array}{l}\text { P3: AAAGCCACGCCTGCAACCAGTG (Fw) } \\
\text { P4: TGGTGGGAGTGCATGGTGTATCTGTG (Rv) }\end{array}$ & 199 & 61 \\
\hline gapdh & $\begin{array}{l}\text { P5: CATCACCATCTTCCAGGAGCGAG (Fw) } \\
\text { P6: CACCCTGTTGCTGTAGCCGAAT (Rv) }\end{array}$ & 754 & 58 \\
\hline
\end{tabular}

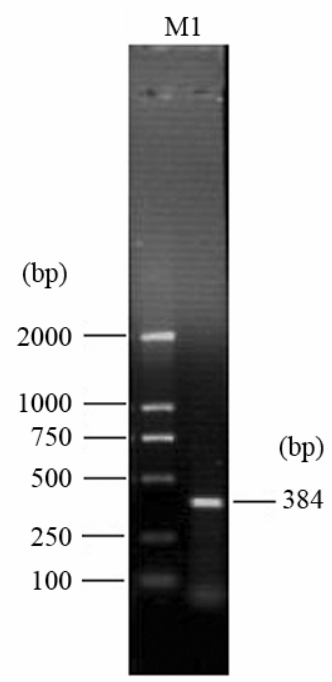

图 1 tnp2基因RT-PCR产物的琼脂糖凝胶电泳

Fig. 1 The RT-PCR products of tnp2 cDNA M: DL2000 DNA 分子量标准; 1: RT-PCR 产物。 M:DL2000 DNA Marker; 1:The RT-PCR products of tnp2 gene.

\section{2 绵羊 $t n p 2$ 基因cDNA核苷酸序列分析及同源性 比较}

在其他物种 $\mathrm{cDNA}$ 已知序列的基础上, 通过 RT-PCR方法将绵羊tnp2的cDNA扩增, 得到了 $384 \mathrm{bp}$ 的tnp2 cDNA编码区核苷酸序列。其中克隆出的部
分ORF长度为 $340 \mathrm{bp}$, 编码 113 个氨基酸残基(图2)。 通过同源性比较, 绵羊tnp2基因cDNA核苷酸序列与 牛(NM_174200)的同源性最高, 达到95.3\%。而与猪 (X57989)、人 (NM_005425)、黑猩猩(XM_ 001141940)、大鼠(NM_017057)和小鼠(NM_013694) 的同源性分别为 $77.2 \% 、 67.4 \% 、 67.1 \% 、 66.7 \%$ 和 $64.9 \%$ 。

\section{3 绵羊雄性生殖细胞体外共培养体系中圆形精 子细胞形成的检测}

用gapdh作为内部参照，对不同培养时间的绵 羊睪丸生殖细胞样品进行tnp2 基因的RT-PCR检测, 得到了 $199 \mathrm{bp}$ 的PCR产物(图 3), 与预期的片段大小 相符。结果显示在 4 月龄及成年睪丸组织和体外共 培养 2-10 周的生殖细胞中均检测到了 tnp2 基因的 转录表达, 但是在肝脏组织中没有检测到tnp2 基因 的转录表达(图 3)。

\section{3 讨 论}

精子发生是从精原细胞分化为雄性配子的复 杂的生物学过程。此过程主要由 3 个阶段组成: 减 数分裂前期阶段，二倍体精原细胞分化为二倍体初 级精母细胞; 减数分裂阶段, 精母细胞经两次连续 减数分裂转变为单倍体圆形精子细胞; 精子形成阶 


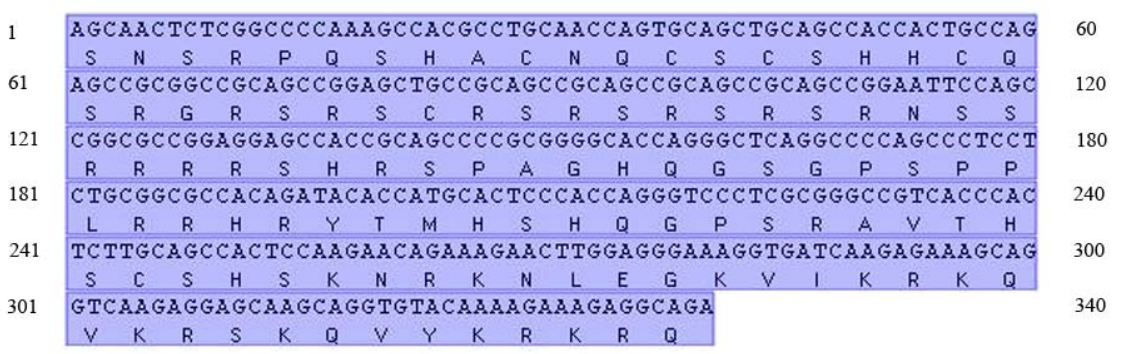

图 2 绵羊 tnp2 cDNA 的核苷酸序列及 113 个氨基酸序列

Fig. 2 The nucleotide sequences of sheep tnp2 gene cDNA and 113 amino acid

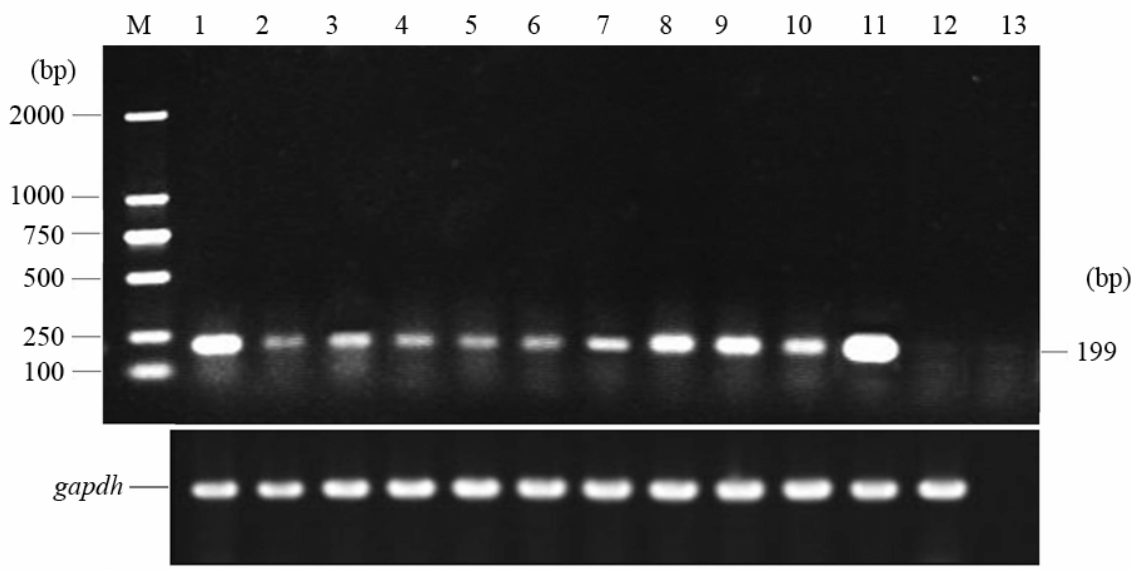

图 3 绵羊睪丸生殖细胞体外共培养系统中tnp2的RT-PCR分析

Fig. 3 The tnp2 RT-PCR analysis of testicular germ cells of sheep co-cultured in vitro

M：DL2000 DNA 分子量标准; 1：4月龄绵羊睪丸组织；2-10：绵羊睪丸生殖细胞与Sertoli细胞体外共培养从第2至第10周的样品；11：成 年绵羊睪丸组织; 12: 绵羊肝组织; 13: 水。

M: DL2000 DNA Marker; 1: Testis tissues of the 4-month sheep; 2-10: Sheep testis germ cells co-cultured with Sertoli cells in vitro from 2nd week to 10th week; 11: Testis tissues of the adult sheep; 12: The sheep liver tissues; $13: \mathrm{H}_{2} \mathrm{O}$.

段, 精子细胞分化为精子。在哺乳动物精子发生过 程中配子核形态和组成均发生了改变, 精原细胞和 精母细胞的染色质蛋白像大多数体细胞一样主要 由组蛋白组成。在减数分裂阶段, 精原细胞和精母 细胞的染色质中可检测到很多新的组蛋白变种。随 后生殖细胞进一步分化进入精子形成阶段, 许多组 蛋白被过渡蛋白替换, 然后顺序性的被鱼精蛋白替 换, 形成精子的核鱼精蛋白(Zhao et al，2004)。在 这个过程中, 染色质发生了转变, 转录活性的核转 变为精子中静止的核, 这需要大量的DNA结合蛋白 的瞬时表达调节。因此, 对于精子发生过程中单倍 体阶段调节基因表达的分子和分子机制的研究是 非常吸引人的。tnp2基因是在雄性生殖细胞减数分 裂后期表达的一个基因, 在精子形成过程各种睪丸 蛋白调控中, 其转录和翻译的调节机制是具有代表 性的。研究发现, 在鼠类中 tnp2基因的mRNA首先 在圆形精子细胞中合成, 以无翻译活性的mRNA核
蛋白颗粒形式储存长达 5 - 7 天, 直至在长型精子细 胞中被激活指导翻译。过渡蛋白2(transition protein-2, TP2)是形成鱼精蛋白进而完成染色质凝 聚所必需的(Meistrich et al, 2003; Zhao et al, 2004)。 为了研究绵羊的精子细胞形成和变态过程, 我们克 隆了绵羊的tnp2基因。

Reinhart et al(1991)报告了牛的tnp2基因和氨基 酸组成（NM_174200），目前绵羊tnp2基因序列尚 未报道。本研究通过RT-PCR和分子克隆技术, 成 功克隆了tnp2基因的cDNA编码区片段, 获得了绵羊 tnp2基因的部分 $\mathrm{cDNA}$ 序列, 为克隆全序列打下基 础。对绵羊和其他哺乳动物的tnp2进行cDNA核苷酸 序列的同源性比较发现，该扩增序列与牛的核苷酸 序列具有 $95.3 \%$ 的同源性。而与猪、人、黑猩猩、 大鼠和小鼠比较也具有较高的同源性。说明 tnp2基 因在进化上具有一定的保守性, 特别是在反刍动物 中具有非常高的保守性。 
目前, 国内外学者相继在小鼠、大鼠和牛等动 物上成功地建立了生殖细胞体外培养的方法, 并利 用体外共培养系统对精子发生过程中相关因子和 基因进行了研究 (Lee et al, 2001; Iwanami et al, 2005; Huleihel et al, 2007)。本研究采用四月龄的 绵羊睪丸进行体外共培养。通过RT-PCR法分析显 示, 此四月龄的睪丸组织已经存在圆形精子细胞 （图3）。对4月龄的睪丸组织制备的共培养体系进 行基因的转录表达分析, 结果显示从 $2-10$ 周一直

\section{参考文献:}

Griswold MD. 1995. Interactions between germ cells and Sertoli cells in the testis[J]. Biol Reprod, 52: 211-216.

Hecht NB. 1990. Regulation of "haploid expressed genes" in male germ cells[J]. J Reprod Fertil, 88: 679-693.

Huleihel M, Abuelhija M, Lunenfeld E. 2007. In vitro culture of testicular germ cells: Regulatory factors and limitations[J]. Growth Factors, 25(4): 236-252.

Iwanami Y, Kobayashi T, Kato M, Hirabayashi M, Hochi S. 2005. Characteristics of rat round spermatids differentiated from spermatogonial cells during co-culture with Sertoli cells, assessed by flow cytometry, microinsemination and RT-PCR [J]. Theriogenology, 65(2): 288-298.

Kierszenbaum AL. 1994. Mammalian spermatogenesis in vivo and in vitro: a partnership of spermatogenic and somatic cell lineages[J]. Endocr Rev, 15: 116-134.

Lee DR, Kaproth MT, Parks JE. 2001. In vitro production of haploid germ cells from fresh or frozen-thawed testicular cells of neonatal bulls[J]. Biology of Reproduction, 65: 873-878.

Marret C, Avallet O, Perrard-Sapori MH, Durand P.1998.Localization and quantitative expression of mRNAs encoding the testis-specific histone TH2B, the phosphoprotein $\mathrm{p} 19$, the transition proteins 1 and 2 during pubertal development and throughout the spermatogenic cycle of the rat [J]. Mol Reprod Dev, 51: 22-35.

Meistrich ML, Mohapatra B, Shirley CR, Zhao M. 2003. Roles of transition nuclear proteins in spermiogenesis[J]. Chromosoma, 111: 483-488. 有tnp2基因的转录表达，表明该共培养体系在体外 长达十周的培养过程中一直存在着生殖细胞的分 化及精子发生过程, 不断地产生圆形精子细胞。这 与我们对绵羊生殖细胞体外共培养系统中细胞形 态的观察结果相一致 (未发表资料)。这为我们利 用该体系来进行绵羊精子发生过程中生殖细胞分 化、减数分裂和圆形精子细胞形成过程的相关因子 和基因的研究奠定了基础。

Parvinen M. 1982. Regulation of the seminiferous epithelium[J]. Endocr Rev, 3: 404-417.

Reinhart N, Kremling H, Luerssen H, Adham IM, Engel W. 1991. Characterization of a gene encoding a basic protein of the spermatid nucleus, TNP2, and its close linkage to the protaminegenes in the bull[J]. Biol Chem Hoppe-Seyler, 372 (6): 431-436.

Skinner MK. 1991. Cell-cell interactions in the testis[J]. Endocr Rev, 12: 45-77.

Wu YJ, Luo FH, Xue XX, Bou S. 2005. Long-term culture and spermatogenisis observation of germ cells from seminiferous tubules of Cashmere goat[J]. Acta Scientiarum Naturalium Universitatis NeiMongol, 36(4): 411-416. [吴应积, 罗奋华, 薛晓先, 旭日干. 2005. 线山羊雄性生殖细胞的长期培养和分化观察. 内蒙古大学学报(自 然科学版), 36(4): 411-416.]

Zhang Y, Luo FH, WU YJ. 2007. Co-culture and spermatogenesis observation of germ cells from rat seminiferous tubules[J]. Chinese Journal of Andrology, 22(12): 39-42. [张 岩, 罗奋华, 吴应积. 2007 大鼠曲细精管生殖细胞体外共培养和精子发生过程的观察. 中国 男科学杂志, 22(12): 39-42.]

Zhao M, Shirley CR, Hayashi S,Marcon L, Mohapatra B, Suganuma R, Behringer RR, Boissonneault G, Yaagimachi R, Meistrich ML. 2004. Transition nuclear proteins are required for normal chromatin condensation an functional sperm development[J]. Genesis, 38: 200-213. 